\title{
Prevalence of Shoulder Pain Disability among Assistive Device Users with Paraplegia
}

\author{
Acharya BD, ${ }^{1}$ Rawal M, ${ }^{2}$ Rokaya $\mathrm{PK}^{2}{ }^{2}$ Karki $\mathrm{D},{ }^{2}$ Limbu $\mathrm{D},{ }^{2}$ Binaya $\mathrm{PL}^{3}$ \\ ${ }^{1}$ Department of Physiotherapy, Karnali Academy of Health Sciences, Jumla, Nepal, ${ }^{2}$ Department of \\ Orthopedics, Karnali Academy of Health Sciences, Jumla, Nepal, ${ }^{3}$ Final Year's Student, Bachelor in \\ Public Health.
}

Corresponding Author:

Bishnu Dutta Acharya

Karnali Academy of Health Sciences, Jumla, Nepal

Email: mugaliaacharya@gmail.com

\begin{abstract}
Introduction: To identify the prevalence of shoulder pain disability in paraplegic patients using assistive devices following Spinal Cord Injury.

Methods: This was a cross-sectional study done among 53 SCI paraplegia patients having shoulder pain after use of assistive devices. Pre-test was done among 5 subjects. The association among different variables with shoulder was tested by using chi-square test. SPSS version 16 was applied to find the result.

Results: The study showed that almost $98.11 \%$ of the respondents had shoulder pain with the assistive device users. Among them all of the participants were using wheelchair as the assistive devices and $86.3 \%$ had mild disability, $11.8 \%$ moderate disability and 2\% sever disability. Among them, $72 \%$ of the patients had stayed in the hospital for less than six months. Shoulder pain was dependent on age, gender, duration of assistive devices used, type of assistive devices used and level of injury

Conclusions: Shoulder pain is common and has a high prevalence rate in both traumatic as well as non-traumatic spinal cord injury. Wheelchair user have more shoulder pain then other assistive devices users. Shoulder pain has a negative effect on activities of daily living and is a potential cause of activity limitations.
\end{abstract}

Keywords: assistive devices; paraplegia; shoulder pain; spinal cord injury.

\section{INTRODUCTION}

Spinal cord injury is defined as any defect in transmission of signals from and to the brain due to any disruption of spinal cord, leading to paralysis below the affected level. Patients with SCI uses wheelchair, cane, crutches and other devices for mobility in the form of assistive devices. ${ }^{1}$ More than $5,00,000$ suffer from SCI each year. ${ }^{2}$ Shoulder pain is any pain in or around the shoulder joint during any functional activity or at rest affecting the daily activities. Inability to perform the daily activity as compared to the normal people of the same age is disability. Prevalence of shoulder pain among SCI is $35.5 \%$ in Motorize wheel chair (MWC), 47.6\% using crutch or canes, and $33.3 \%$ without assistance. ${ }^{3}$
The estimate incidence of traumatic SCI in Nepal was 00-5000 and prevalence 1500-25000 in 2007. SIRC is the specific organization for SCI rehabilitation center, located in Banepa which is $23 \mathrm{~km}$ east from Kathmandu Valley. Number of SCI patients at SIRC was 123 in the year 2013. Timely intervention and proper uses of mobility technique helps to reduce shoulder pain. ${ }^{5}$ Without intervention, shoulder pain after SCI is associated with additional losses in function and community mobility. ${ }^{3}$

However in context of Nepal and at SIRC though prevalence of SCI has been estimated, but shoulder pain and disability due to use of assistive devices is unknown till date. Immobility in SCI due to 
shoulder pain reduce the quality of life and physical activity, which leads to significant psychological consequences, secondary morbidities and reduce the person's ability and motivation.

\section{Conceptual Framework}

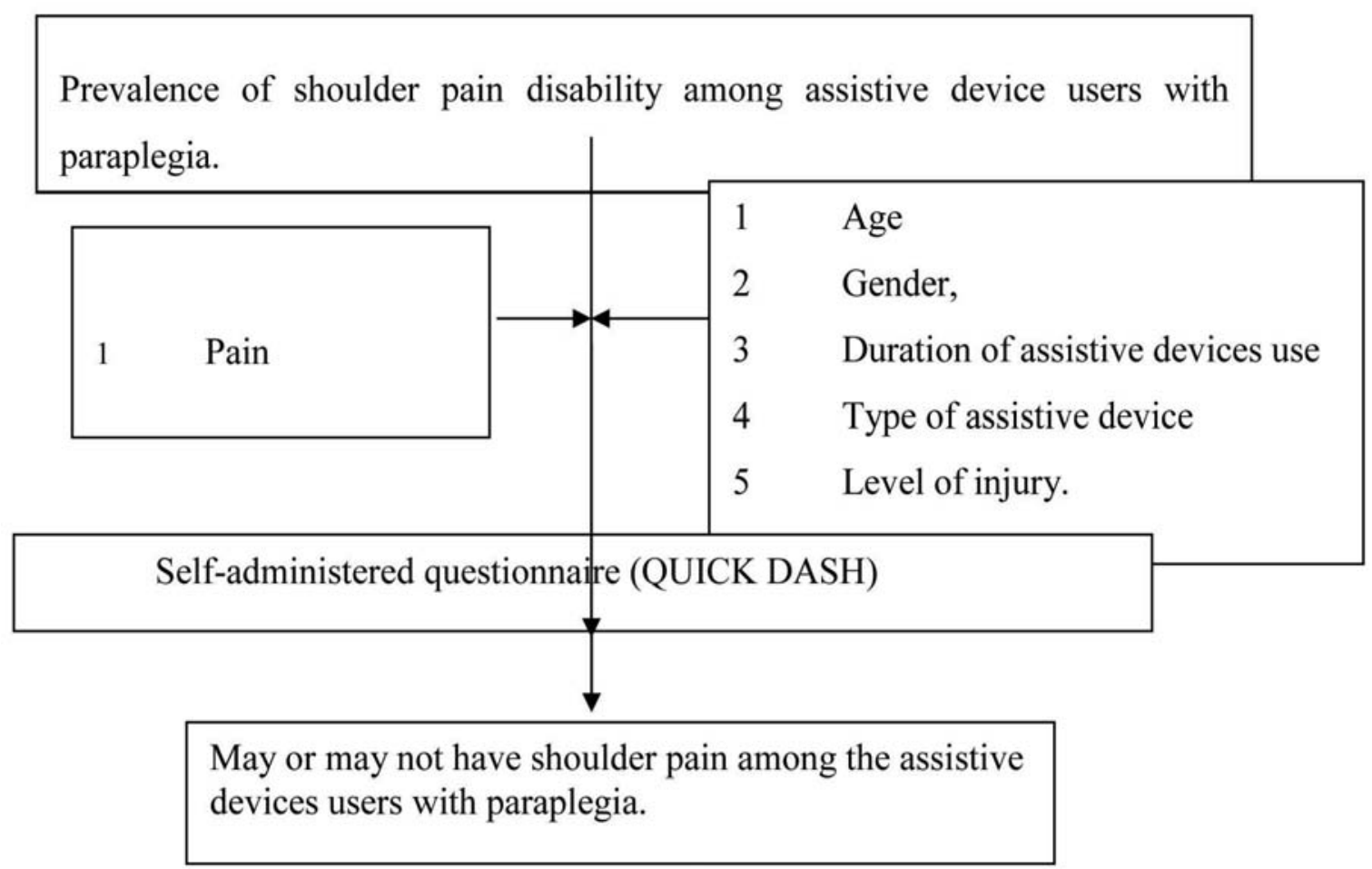

Figure 1. Conceptual framework for Prevalence of shoulder pain disability among assistive device users with paraplegia.

\section{METHODS}

A descriptive, cross-sectional study was used as the study aim to describe the state of affairs and was done for certain period of time with quantities method. The study site was taken at Spinal Injury Rehabilitation Centre, Banepa. It is the only specific rehabilitation center in central developmental region for SCI and most are referred to these centers. The targeted population were all SCI patient using assistive devices with paraplegia coming at SIRC.

The inclusion criteria were paraplegic SCI patients, SCI patients with shoulder pain, able to understand the consent, using any assistive devices and having the shoulder pain for more than 3 weeks. Were as the exclusion criteria were with no pain, use of NSAID and cortisone, bed ridden and with any recent shoulder surgery with in last year.

Non-probability purposive Sampling was used with sample size of 53 . 


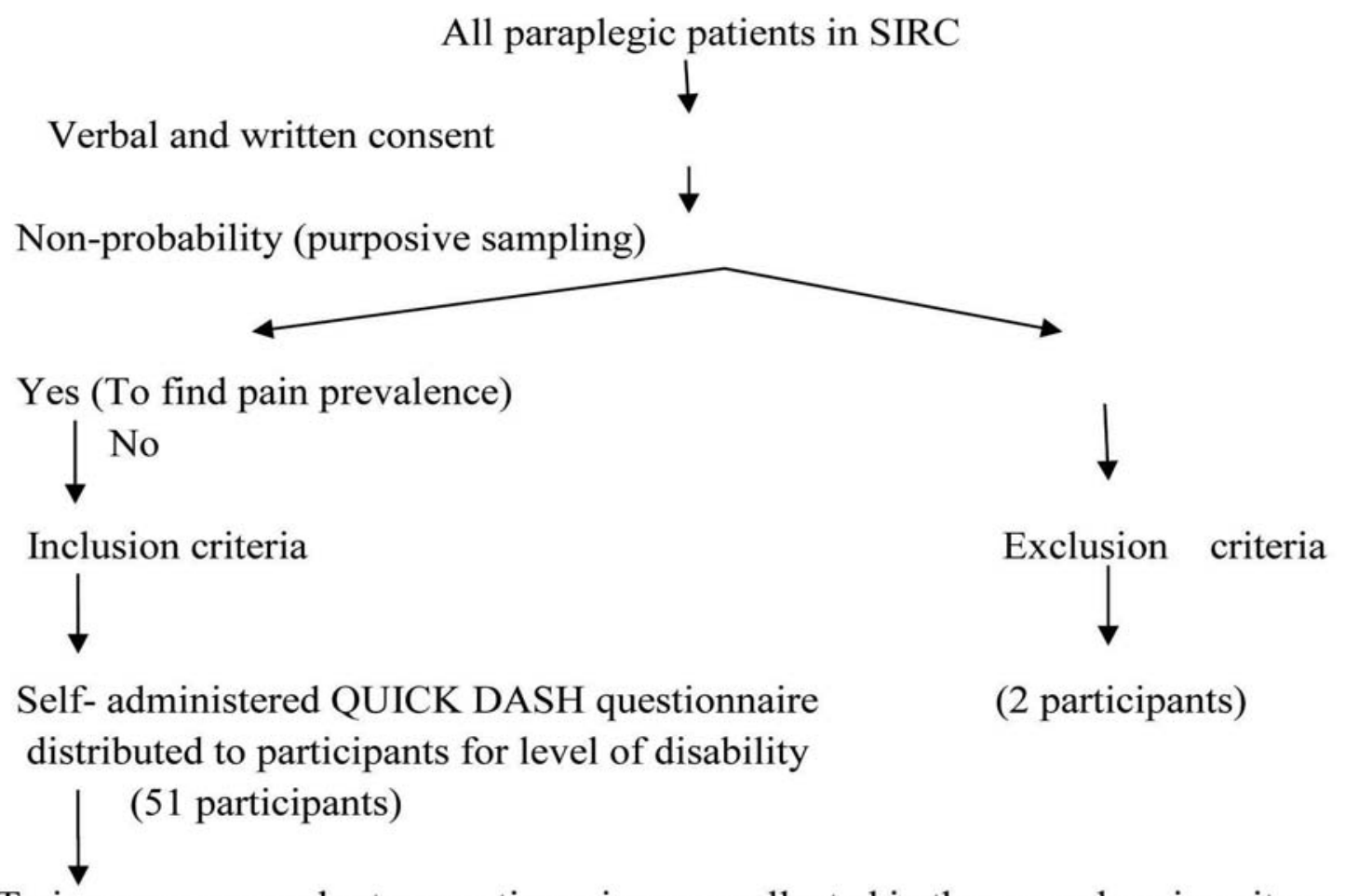

To increase respond rate, questionnaire was collected in the same day since it would not have taken more than $15 \mathrm{~min}$.

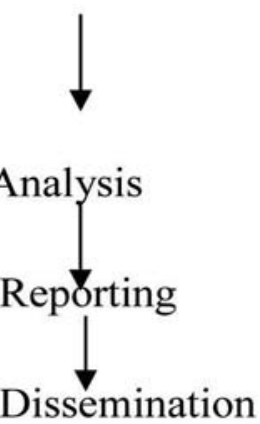

Figure 2. Showing the sampling process.

The study measurement was divided into two variables i.e. dependent and independent variables. The independent variables were age, gender, duration of assistive device used, type of assistive devices and level of injury whereas the dependent variable was only shoulder pain. The data were collected by demographic details and the tool QUICK DASH. The procedure was explained to participants. The Psychometric properties of quick DASH reliability 0.94 , validity $=\beta 0.70$ and responsiveness $=0.79$. Pre-test was done after the permission from the KUIRC among five paraplegia SCI patients having the shoulder pain i.e. $10 \%$ of total sample size. The collected data were checked and inter into SPSS-16.0 for the analysis. Chi-square test was performed to determine an association between dependent and independent variables.

\section{Statistical Testing}

Descriptive statistics such as percentage, mean, 
median and standard deviation was calculated.

Chi square test was used to find out the association between shoulder pain and selected variables like: age, gender, duration of assistive devices uses, level of injury, type of assistive device.

To evaluate the severity of shoulder pain:

$\begin{array}{ll}\text { Severe pain } & >66.66 \% \\ \text { Moderate pain } & >33.3 \%-<66.66 \% \\ \text { Mild Pain } & <33.3 \%\end{array}$

An official permission was taken from KUIRC and from the SIRC rehabilitation center. Verbal and written consent was taken from all subject prior to data collection.

\section{DISCUSSION}

This descriptive cross-sectional study using purposive sampling technique was conducted to assess the prevalence of shoulder pain among the assistive device users with paraplegia with spinal cord injury at SIRC. The discussion was made by reviewing different literature on Prevalence of Shoulder Pain and Disability Among Assistive Devices Users with Paraplegia. Spinal cord injury is a devastating, sudden onset injury which may result in permanent paralysis and loss of physical function. The injured individual is usually dependent on a wheelchair for mobility and may require varying levels of personal care assistance with activities of daily living.

Medical treatment and rehabilitation following spinal cord injury typically requires a lengthy period of hospitalization and rehabilitation. Literature confirms that, SCI survivors who use manual wheelchair has a high prevalence of shoulder pain and it effect on daily activities, work, and participation in recreational activities. In our study the shoulder pain prevalence is 98.11\% which show that the wheelchair users have high prevalence as the previous literature supports. In my study, all the participants were using the wheelchair that also shows the more rate of shoulder pain.

The present study shows that the almost all the participants had shoulder pain who use assistive devices for the locomotion. There were total 53 of the participants in our study and 51 had shoulder pain and 2 without pain all the 53 participants were using the manual wheelchair for mobility. This giving is similar with the study conducted in Sweden which showed shoulder pain as a common problem among paraplegic patients who uses wheelchair as an assistive device. ${ }^{7}$ Whereas another study conducted in America shows that shoulder pain is third most common pain after legs and back among the SCI using the assistive devices ${ }^{1}$.This study also reveals that shoulder pain prevalence is more in male $94.1 \%$ then female $5.9 \%$ is shown due to involvement of more male population for more challenge household works, involvement in driving and climbing the high cliffs. In our study SCI is common in the active age and in younger population from the age of 10 years to 50 years. In younger population this shows more SCI. This people are more tend to have shoulder pain in the future as they will have to use the wheelchair for longer period of life. M. Alm also had found that pain tendency increase with ongoing uses of wheelchair as age goes on. ${ }^{7}$

In our study stratified by the use of assistive mobility devices all the participants were found to use of wheelchair then other assistive devices. This doesn't support the fact of shoulder pain is $46.7 \%$ in motorized wheelchair users, $35.4 \%$ of manual wheelchair users and $47.6 \%$ of participants using aids such as cane or crutches and $33.3 \%$ of the participants walking without assistance in the study conducted by N.B.Jain et.al.In our study the incidence was more of fall then of other cause for SCI.

According to WHO in south east Asia in the year 2012 the incidence of SCI due to fall was $40 \%$ and $70 \%$ MVA where as in this study were $82.4 \%$ fall and only $5.9 \%$ of them were MVA. This might be due to fact that farming and climbing is the main occupation in context of Nepal. Similar static has been show by the M.K Ranabhat in the green pasture hospital in the year 2007 has also shown that $60 \%$ were from fall and $22 \%$ were of motor vehicle accidents.

The study had some limitation as it cannot be generalized to all the patients with SCI as it is done only in one specific center located at central development region of Nepal. As the study duration is 
shorter so sample size can be limited. Respondent bias as participants might have answered something that is morally right than their true answer.

The study will be implicated among with assistive device users have to fully depend on the upper extremity for locomotion. Shoulder joint is not meant for the weight bearing. So here is high prevalence of shoulder pain among the assistive devices users, especially among the wheelchair users. In order to reduce the shoulder pain among the patients with SCI the physiotherapist who are under the care need to focus primarily on following things:

1. Wheelchair modification is also suggestive to reduce the shoulder pain.

2. Prioritize the treatment on strengthening the upper extremity, changes on the wheelchair as per the participant's geographical variation.

3. Further generalizing study is required for exact prevalence of shoulder pain in larger population or subjects with SCI Nepal.

\section{CONCLUSIONS}

The Shoulder pain is common, and has a high prevalence rate among the spinal cord injury. Participants with wheelchair user have reveled maximum shoulder pain. Shoulder pain has a negative effect on activities of daily living and is a potential cause of activity limitations. The findings indicate a need to develop programs of prevention, patient education, diagnosis and early treatment.

\section{Conflict of Interest: None}

\section{REFERENCES}

1. Nitin B. Jain Laurence D. Association of Shoulder Pain with the Use of Mobility Devices in Persons with Chronic Spinal Cord Injury. PMR author script 2(10): 896-900

2. WHO. International perspective on spinal cord injury-2013
3. Sara J. Mulroy, Lilli Thompson, Bryan Kemp, Patricia Pate Hatchett, Craig J. Newsam. Strengthening and Optimal Movements for Painful Shoulders (STOMPS) in Chronic Spinal Cord Injury: A Randomized Controlled Trial. Physical Therapy-2011. Volume 91 Number 3

4. Carol Y Scovi, Manoj K Ranabhat, Iain B Craighead: Follow-up study of spinal cord injured patients after discharge from inpatient rehabilitation in Nepal in 2007. Published in Spinal Cord 2012;50, 232237

5. Ludewig Christine M Wilson, Benjamin A Howe and Paula M Deborah A Nawoczenski, Jordan M Ritter-Soronen. Clinical trial of exercise of shoulder pain in chronic spinal cord injury ; Physical therapy;2006; 86:1604-1618

6. Essi Khamis, Jadallah M. Shoulder Pain among Rehabilitated Spinal Cord Injured Persons Using Manually Propelled Wheelchairs in the Gaza Strip: Asia Pacific disability rehabilitation journal, 2012 Vol 23, No.2

7. Marie Alm, Helena Saraste and Cecilia Norrbrink. shoulder pain persons with thoracic spial cord injury: prevalence and characteristic. Journal Rehab Med .2008; 40: 277-283

8. Giorgio Ferriero a, Andrea Giordano b, Francesco Sartorio, Stefano Vercelli a, Elisa Brigatti c . Psychometric properties of Quick DASH. A classical test theory and Rasch analysis study. Manual Therapy (2011) 